\title{
Health-related quality of life of postmenopausal women with hormone receptor-positive, human epidermal growth factor receptor 2-negative advanced breast cancer treated with ribociclib + letrozole: results from MONALEESA-2
}

\author{
Sunil Verma ${ }^{1}$ D . Joyce O'Shaughnessy ${ }^{2} \cdot$ Howard A. Burris $^{3} \cdot$ Mario Campone $^{4} \cdot$ Emilio Alba $^{5} \cdot$ David Chandiwana $^{6}$. \\ Anand A. Dalal ${ }^{6} \cdot$ Santosh Sutradhar ${ }^{6} \cdot$ Mauricio Monaco $^{6} \cdot$ Wolfgang Janni $^{7}$
}

Received: 28 February 2018 / Accepted: 26 March 2018 / Published online: 13 April 2018

(C) The Author(s) 2018

\begin{abstract}
Purpose Evaluate patient-reported outcomes (PROs) for postmenopausal women with hormone receptor-positive (HR+), human epidermal growth factor receptor 2-negative (HER2-) advanced breast cancer treated with first-line ribociclib plus letrozole.

Methods In the phase III MONALEESA-2 study (NCT01958021), 668 patients were randomized 1:1 to ribociclib (600 mg/ day; 3-weeks-on/1-week-off) plus letrozole (2.5 mg/day) or placebo plus letrozole. PROs were assessed using the European Organisation for Research and Treatment of Cancer core quality-of-life (EORTC QLQ-C30) and breast cancer-specific (EORTC QLQ-BR23) questionnaires. Changes from baseline and time to deterioration in health-related quality of life (HRQoL) were analyzed using linear mixed-effect and stratified Cox regression models, respectively. Exploratory analysis of area-under-the-curve for change from baseline in pain score (AUC-pain) was performed.

Results On-treatment HRQoL scores were consistently maintained from baseline and were similar between arms. A clinically meaningful ( $>5$ points) reduction in pain score was observed as early as Week 8 and was maintained up to Cycle 15 in the ribociclib arm. A statistically significant increase in mean AUC-pain was also observed in the ribociclib arm. Scores for all other EORTC QLQ-C30 and EORTC QLQ-BR23 domains were maintained from baseline and were similar between arms. Conclusions HRQoL was consistently maintained from baseline in postmenopausal women with HR+, HER2- advanced breast cancer receiving ribociclib plus letrozole and was similar to that observed in the placebo plus letrozole arm. Together with the improved clinical efficacy and manageable safety profile, these PRO results provide additional support for the benefit of ribociclib plus letrozole in this patient population.
\end{abstract}

Keywords CDK4/6 inhibitor - Ribociclib · Advanced breast cancer · Health-related quality of life $\cdot$ Endocrine therapy · Hormone receptor-positive

Sunil Verma

DrSunil.Verma@ahs.ca

1 Department of Oncology, Cumming School of Medicine, Tom Baker Cancer Centre, University of Calgary, 1331 29th Street NW, Calgary, AB T2N 4N2, Canada

2 Texas Oncology-Baylor Charles A. Sammons Cancer Center, Baylor University Medical Center, and The US Oncology Network, 3410 Worth Street, Suite 400, Dallas, TX 75246, USA

3 Drug Development, Sarah Cannon Research Institute, 250 25th Avenue North, Suite 100, Nashville, TN 37203, USA
4 Medical Oncology, Institut de Cancérologie de l'Ouest/René Gauducheau Centre de Recherche en Cancérologie, Boulevard Jacques Monod, Nantes 44805, Saint-Herblain, France

5 Medical Oncology Unit, Hospital Universitario Virgen de la Victoria, IBIMA, 29010 Málaga, Spain

6 Novartis Pharmaceuticals Corporation, One Health Plaza, East Hanover, NJ 07936-1080, USA

7 Department of Gynecology, Universitätsklinikum Ulm, Prittwitzstraße 43, 89075 Ulm, Germany 


\section{Introduction}

Ribociclib is an orally bioavailable, highly selective inhibitor of cyclin-dependent kinases (CDK) 4 and 6 (CDK4/6) [1]. In clinical studies, ribociclib has demonstrated significant activity together with endocrine therapy as a first-line treatment in hormone receptor-positive (HR+), human epidermal growth factor receptor 2-negative (HER2-) advanced breast cancer [2-4]. In the phase III MONALEESA-2 study, first-line treatment with ribociclib plus letrozole significantly prolonged progression-free survival (PFS) at the pre-planned interim analysis (hazard ratio: 0.556 ; 95\% confidence interval [CI] 0.429-0.720; $p=0.00000329$ ), showing higher overall response rates versus placebo plus letrozole in postmenopausal women with HR+, HER2- recurrent/metastatic breast cancer [4, 5]. An updated analysis demonstrated maintained treatment benefit with ribociclib plus letrozole: median PFS was 25.3 months versus 16.0 months in the placebo plus letrozole arm (hazard ratio: 0.568; 95\% CI 0.457-0.704; $p=0.0000000963)$ [6].

Targeted combination therapies are associated with higher response rates and delayed progression in patients with HR+, HER2- advanced breast cancer when compared with single-agent endocrine therapy, but this approach can expose patients to additional treatmentrelated toxicities, which can affect their quality of life (QoL) [7]. In general, CDK4/6 inhibitor-based regimens are associated with predictable and manageable safety profiles, with myelosuppression observed most commonly [4, $6,8]$. Recent guidelines recommend that the impact of treatment on QoL should be considered in addition to efficacy and safety [9]. In this analysis of the MONALEESA-2 study, we report validated patient-reported outcomes (PROs) results, including health-related QoL (HRQoL) and improvement in symptoms.

\section{Methods}

\section{Study design and treatment}

A detailed study design has previously been reported [5]. MONALEESA-2 is an ongoing, double-blind, randomized phase III study of first-line ribociclib $(600 \mathrm{mg} /$ day on a 3 -weeks-on/1-week-off schedule) or placebo in combination with letrozole $(2.5 \mathrm{mg} /$ day on a continuous schedule). PROs were a secondary objective. The trial protocol was reviewed and approved by an independent ethics committee and institutional review board at each site. A steering committee oversaw the conduct of the study in conformation with the approved protocol. The study was conducted in accordance with the International Conference on Harmonisation's Harmonised Tripartite Guideline for Good Clinical Practice, all applicable local regulations, and the ethical principles of the Declaration of Helsinki.

\section{PRO assessments}

PRO measures of HRQoL, functioning, disease symptoms, and treatment-related side effects were assessed using the European Organisation for Research and Treatment of Cancer core quality-of-life (EORTC QLQ-C30; v3.0) [10] and breast cancer-specific (EORTC QLQ-BR23; v1.0) questionnaires [11].

Patients were asked to complete both questionnaires at the beginning of each visit at screening, every 8 weeks for the first 18 months, then every 12 weeks thereafter until disease progression, death, loss to follow-up, or withdrawal of consent, and at treatment discontinuation. Questionnaire responses were converted to a score ranging from 0 to 100 . For functional and global health status/QoL scales, a higher numerical score represents a better level of functioning/ HRQoL; a positive change from baseline was considered an improvement in functioning/HRQoL. For symptomatic scales, a higher numerical score represents greater symptom severity; a negative change from baseline was considered an improvement in symptom severity.

\section{Statistical analyses}

PRO analyses were based on the full analysis set ( $N=668)$, following the intent-to-treat principle. For partially completed multi-item scales, scores were equal to the average of the completed items for that particular respondent. Changes from baseline were analyzed using a linear mixed-effect model. Evaluable patients had baseline scores and at least one non-missing postbaseline PRO assessment. A post hoc analysis of time to definitive deterioration (TTD) in overall HRQoL EORTC QLQC30 scores by $\geq 10 \%$ was performed for patients with, versus without, a PFS event, within each treatment arm, and among all treated patients across both arms using the stratified log-rank test with a two-sided $p$-value. A definitive deterioration event was defined as a decrease of $\geq 10 \%$ in EORTC QLQ-C30 global health status/QoL score relative to baseline, with no subsequent improvement above this threshold, or death due to any cause. Patients with no definitive deterioration events were censored at the date of the last available PRO assessment. HRQoL deterioration was considered clinically meaningful using previously established thresholds for minimally important differences (MID) in QoL; for EORTC QLQC30, the threshold for MID was a change of 5-10 points from baseline [12]. The Kaplan-Meier method was used 
to estimate the median TTD in HRQoL by $\geq 10 \%$; hazard ratio and two-sided 95\% CIs were estimated using a stratified Cox regression model. Exploratory analysis of area-under-the-curve (AUC) for change from baseline in EORTC QLQ-C30 pain scores (AUC-pain) was also performed; mean AUC-pain was compared between the two treatment arms using a t-test. No multiplicity adjustments were made for $p$-values for exploratory and subgroup analyses.

\section{Results}

\section{Patient characteristics and disposition}

A total of 668 patients were randomized to ribociclib (600 mg/day on a 3-weeks-on/1-week-off schedule) plus letrozole $(2.5 \mathrm{mg} /$ day on a continuous schedule; $n=334)$ or placebo plus letrozole $(n=334)$ [4]. Baseline patient and disease characteristics were well balanced across treatment arms (Table 1).

Measurable disease data were based on a cut-off date of January 2, 2017. Patient demographics, disposition, and EORTC questionnaire completion data were based on a cut-off date of January 29, 2016. All PRO data were based on a cut-off date of January 4, 2017.

\section{EORTC QLQ-C30 global health status/QoL scale}

Overall compliance rates of patients completing the HRQoL questionnaires during the treatment period were high in both treatment arms (Table 2).

EORTC QLQ-C30 global health status/QoL scores were consistently maintained from baseline and were similar in both treatment arms during the study treatment period, with clinically meaningful ( $>5$ points from baseline) improvements observed at some timepoints (Fig. 1). Differences between treatment arms in overall HRQoL were less than the MID. TTD by $\geq 10 \%$ in overall HRQoL was also similar between treatment arms (hazard ratio: 0.944; 95\% CI 0.720-1.237) [13]. Mean overall HRQoL worsened in both treatment arms at end of treatment (EOT) despite the earlier improvements from baseline (Fig. 1). Overall HRQoL was also maintained from baseline in both treatment arms during the study treatment period in subgroups of patients with bone-only metastases, visceral disease, those with a best overall response of complete response (CR) or partial response (PR; data not shown), and those with an Eastern Cooperative Oncology Group performance status of 0 or 1 (Fig. 2).

According to post hoc analyses, a significantly greater delay in TTD ( $\geq 10 \%$ decrease) in overall HRQoL was observed in patients who did not experience a PFS event versus those who did experience a PFS event (disease
Table 1 Patient demographics and baseline characteristics [4]

\begin{tabular}{|c|c|c|}
\hline Patient/baseline characteristic & $\begin{array}{l}\text { Ribociclib + letrozole } \\
n=334\end{array}$ & $\begin{array}{l}\text { Placebo + letrozole } \\
n=334\end{array}$ \\
\hline Median age, years (range) & $62(23-91)$ & $63(29-88)$ \\
\hline \multicolumn{3}{|l|}{ Race, $n(\%)$} \\
\hline White & $269(80.5)$ & $280(83.8)$ \\
\hline Asian & $28(8.4)$ & $23(6.9)$ \\
\hline Black & $10(3.0)$ & $7(2.1)$ \\
\hline Other/unknown & $27(8.1)$ & $24(7.2)$ \\
\hline \multicolumn{3}{|l|}{ ECOG PS, $n(\%)$} \\
\hline 0 & $205(61.4)$ & $202(60.5)$ \\
\hline 1 & 129 (38.6) & $132(39.5)$ \\
\hline \multicolumn{3}{|l|}{ Metastatic sites, $n(\%)$} \\
\hline Visceral disease & $197(59.0)$ & $196(58.7)$ \\
\hline Bone-only disease & $69(20.7)$ & $78(23.4)$ \\
\hline De novo metastatic disease, $n(\%)$ & $114(34.1)$ & $113(33.8)$ \\
\hline \multicolumn{3}{|l|}{ Prior (neo)adjuvant therapy, $n(\%)^{\mathrm{a}}$} \\
\hline Chemotherapy & $146(43.7)$ & $145(43.4)$ \\
\hline Endocrine therapy ${ }^{\mathrm{b}}$ & $175(52.4)$ & $171(51.2)$ \\
\hline
\end{tabular}

Data cut-off: January 29, 2016

ECOG PS Eastern Cooperative Oncology Group performance status

${ }^{a}$ Some patients received both chemotherapy and endocrine therapy as neoadjuvant or adjuvant treatment

${ }^{b}$ Endocrine therapy includes anastrozole, exemestane, goserelin, letrozole, tamoxifen, and treatments coded as "other" 
Table 2 EORTC QLQ-C30 and EORTC QLQ-BR23 questionnaire completion rates

\begin{tabular}{|c|c|c|c|c|}
\hline & \multicolumn{2}{|c|}{$\begin{array}{l}\text { Ribociclib + letrozole } \\
n=334\end{array}$} & \multicolumn{2}{|l|}{$\begin{array}{l}\text { Placebo+ letrozole } \\
n=334\end{array}$} \\
\hline & $\begin{array}{l}\text { Patients on study } \\
\text { at scheduled day, } \\
n^{\mathrm{a}}\end{array}$ & $\begin{array}{l}\text { Patients on study at scheduled day with } \\
\text { valid questionnaire within time window, } \\
n(\%)^{\mathrm{b}}\end{array}$ & $\begin{array}{l}\text { Patients on study } \\
\text { at scheduled day, } \\
n^{\mathrm{a}}\end{array}$ & $\begin{array}{l}\text { Patients on study at scheduled day with } \\
\text { valid questionnaire within time window, } \\
n(\%)^{\mathrm{b}}\end{array}$ \\
\hline \multicolumn{5}{|c|}{ EORTC QLQ-C30 completion rates } \\
\hline Baseline & 334 & $324(97.0)$ & 334 & $327(97.9)$ \\
\hline Cycle 3 Day 1 & 309 & $293(94.8)$ & 298 & $291(97.7)$ \\
\hline Cycle 5 Day 1 & 283 & $269(95.1)$ & 273 & $264(96.7)$ \\
\hline Cycle 7 Day 1 & 268 & $257(95.9)$ & 259 & $255(98.5)$ \\
\hline Cycle 9 Day 1 & 248 & $237(95.6)$ & 236 & $227(96.2)$ \\
\hline Cycle 11 Day 1 & 236 & $230(97.5)$ & 215 & $202(94.0)$ \\
\hline Cycle 13 Day 1 & 216 & $206(95.4)$ & 195 & $186(95.4)$ \\
\hline Cycle 15 Day 1 & 171 & $154(90.1)$ & 136 & $133(97.8)$ \\
\hline Cycle 17 Day 1 & 118 & $110(93.2)$ & 89 & $81(91.0)$ \\
\hline Cycle 19 Day 1 & 69 & $61(88.4)$ & 45 & $41(91.1)$ \\
\hline Cycle 22 Day 1 & 20 & $17(85.0)$ & 17 & $13(76.5)$ \\
\hline Cycle 25 Day 1 & 4 & $3(75.0)$ & - & - \\
\hline \multicolumn{5}{|c|}{ EORTC QLQ-BR23 completion rates } \\
\hline Baseline & 334 & $324(97.0)$ & 334 & $326(97.6)$ \\
\hline Cycle 3 Day 1 & 308 & $294(95.5)$ & 298 & $289(97.0)$ \\
\hline Cycle 5 Day 1 & 283 & $269(95.1)$ & 273 & $265(97.1)$ \\
\hline Cycle 7 Day 1 & 268 & $257(95.9)$ & 259 & $254(98.1)$ \\
\hline Cycle 9 Day 1 & 248 & $237(95.6)$ & 236 & $228(96.6)$ \\
\hline Cycle 11 Day 1 & 236 & $230(97.5)$ & 215 & $203(94.4)$ \\
\hline Cycle 13 Day 1 & 216 & $206(95.4)$ & 195 & $184(94.4)$ \\
\hline Cycle 15 Day 1 & 171 & $153(89.5)$ & 136 & $131(96.3)$ \\
\hline Cycle 17 Day 1 & 118 & $110(93.2)$ & 89 & $80(89.9)$ \\
\hline Cycle 19 Day 1 & 69 & $61(88.4)$ & 45 & $42(93.3)$ \\
\hline Cycle 22 Day 1 & 20 & $17(85.0)$ & 17 & $13(76.5)$ \\
\hline Cycle 25 Day 1 & 4 & $3(75.0)$ & - & - \\
\hline
\end{tabular}

EORTC QLQ-BR23 European Organisation for Research and Treatment of Cancer breast cancer-specific questionnaire, EORTC QLQ-C30 European Organisation for Research and Treatment of Cancer core quality-of-life questionnaire, $Q o L$ quality of life

${ }^{a}$ Number of patients eligible to complete the questionnaire at the corresponding visit

${ }^{\mathrm{b}}$ At least one valid score among QoL, physical functioning, emotional functioning, and social functioning scores was required for the EORTC QLQ-C30 questionnaire

progression or death; Fig. 3). Delay in HRQoL deterioration was observed in both the ribociclib plus letrozole arm (hazard ratio: $0.59 ; 95 \%$ CI 0.39-0.87; $p=0.008$; Fig. 3a) and placebo plus letrozole arm (hazard ratio: $0.41 ; 95 \% \mathrm{CI}$ $0.26-0.63 ; p=0.000031$; Fig. 3b). The delay in HRQoL deterioration in patients without, versus with, a PFS event was similar in the population of all treated patients (hazard ratio: 0.50 ; 95\% CI 0.38-0.66; $p=0.000000943$; Fig. 3c).

\section{EORTC QLQ-C30 symptom scales}

Mean baseline scores for EORTC QLQ-C30 symptoms, including fatigue [30.9 (standard deviation [SD]: 23.9) versus 31.4 (SD: 24.2) in the ribociclib versus placebo arms, respectively], nausea and vomiting [7.3 (SD: 15.3) versus 8.6 (SD: 17.8)], and diarrhea [8.1 (SD: 16.8) versus 7.1 (SD: 16.4)] were generally at the lower end of the score range in both treatment arms, indicating lower symptom severity.

During study treatment, HRQoL was maintained in patients experiencing fatigue, nausea and vomiting, and diarrhea; no clinically relevant differences in change from baseline of EORTC QLQ-C30 global health status/QoL score deterioration were observed in patients with these symptoms (data not shown). Although symptom scores were generally higher in the ribociclib plus letrozole arm during treatment and at EOT, the mean changes from baseline were less than the MID. Similar results were observed for additional EORTC QLQ-C30 questionnaire domains, including 


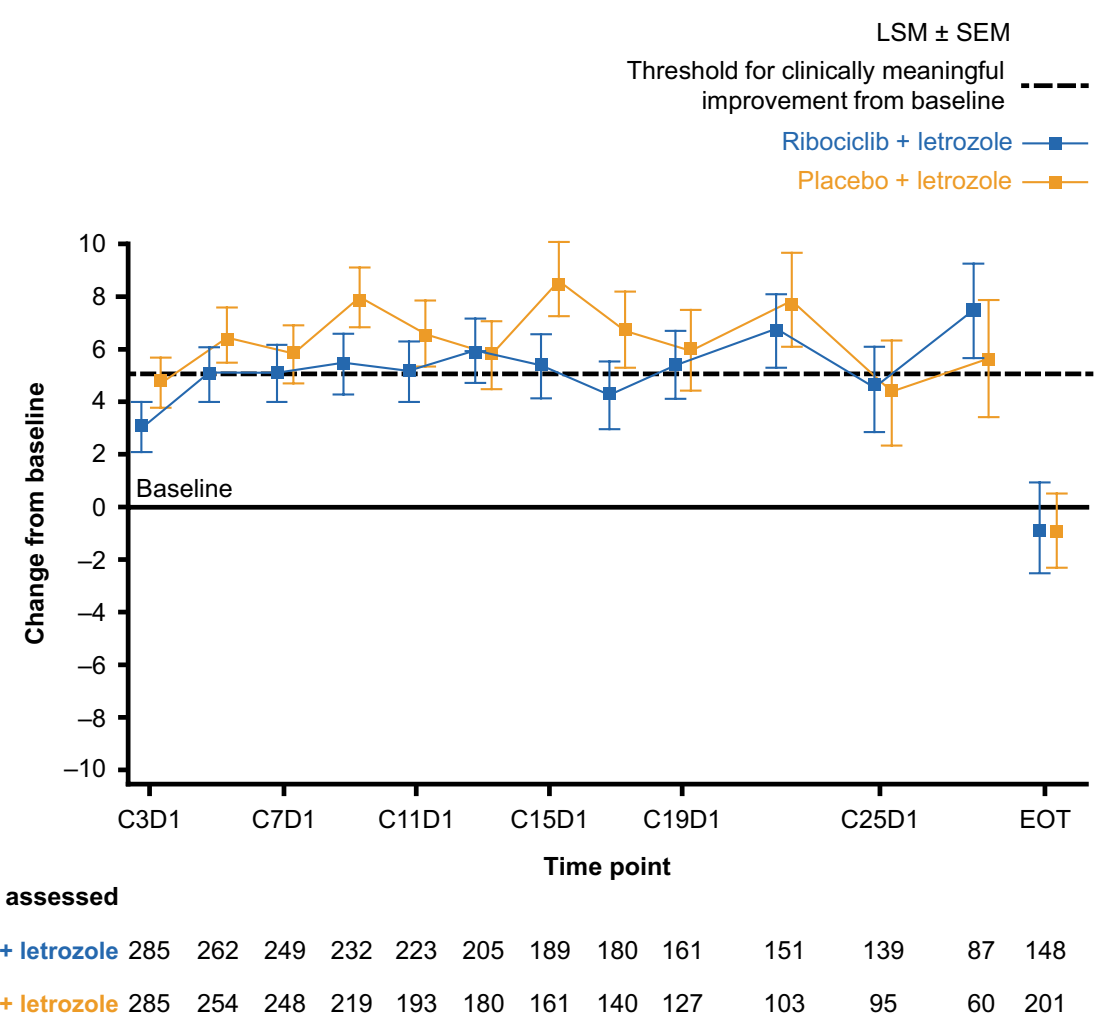

Fig. 1 Overall change from baseline in patient-reported EORTC QLQ-C30 global health status/QoL scores by treatment. $C$ Cycle, $D$ Day, EORTC QLQ-C30 European Organisation for Research and Treatment of Cancer core quality-of-life questionnaire, EOT end of treatment, $H R Q o L$ health-related quality of life, $L S M$ least squares mean, $Q o L$ quality of life, $S E M$ standard error of the mean. Changes

physical, emotional, cognitive, and social functioning and for breast cancer-specific EORTC QLQ-BR23 questionnaire domains, including future perspective, side effects, and upset by hair loss (Table 3 ).

As reported previously, a clinically meaningful ( $>5$ points) reduction from baseline in EORTC QLQ-C30 pain score was observed as early as Week 8 in the ribociclib arm [14]. This clinically meaningful reduction in pain score was maintained up to Cycle 15 in the ribociclib plus letrozole arm (Fig. 4). Improvements of $>5$ points from baseline in pain score were only observed in the placebo plus letrozole arm at Cycles 7 and 15; during all other cycles, the improvement was $\leq 5$ points (Fig. 4). An improvement in pain score was also observed in the ribociclib plus letrozole arm for specific patient subgroups, including those with bone-only metastases, visceral disease (data not shown), and patients with a best overall response of CR or PR (Fig. 5).

As conventional longitudinal analysis of PRO endpoints may not always capture the totality of the benefit throughout the treatment period, an exploratory AUC analysis for change from baseline in EORTC QLQ-C30 pain score was performed to characterize changes in pain during treatment. from baseline in patient-reported EORTC QLQ-C30 global health status/QoL scores were determined using a linear mixed-effect model. Positive changes from baseline indicate improvement in HRQoL. A $>$ 5-point improvement from baseline in HRQoL score was defined as clinically meaningful. Data cut-off: January 4, 2017

A reduced numeric score versus baseline represented less pain severity, whereas an increased pain score indicated greater pain severity [15]. According to the exploratory AUC analysis, a statistically significant reduction in the mean AUC-pain was observed in all patients (mean difference: $-1952 ; 95 \%$ CI $-3826,-79 ; p=0.0412$ ) and subgroups of patients with measurable disease at baseline (mean difference: -2273 ; 95\% CI $-4332,-214 ; p=0.0306$ ) in the ribociclib plus letrozole arm compared with the placebo plus letrozole arm, confirming the improvement from baseline in EORTC QLQ-C30 pain score and indicating reduced pain severity (Fig. 6).

\section{Discussion}

Several studies have assessed the QoL of patients diagnosed with breast cancer, yet few have investigated QoL in the setting of recurrent/metastatic disease [16]. Evaluation of the impact of treatment modalities on QoL in patients with advanced disease is also limited. This study presented 
(a)

$\mathrm{LSM} \pm \mathrm{SEM}$

Threshold for clinically meaningful improvement from baseline

Ribociclib + letrozole —-

Placebo + letrozole —-

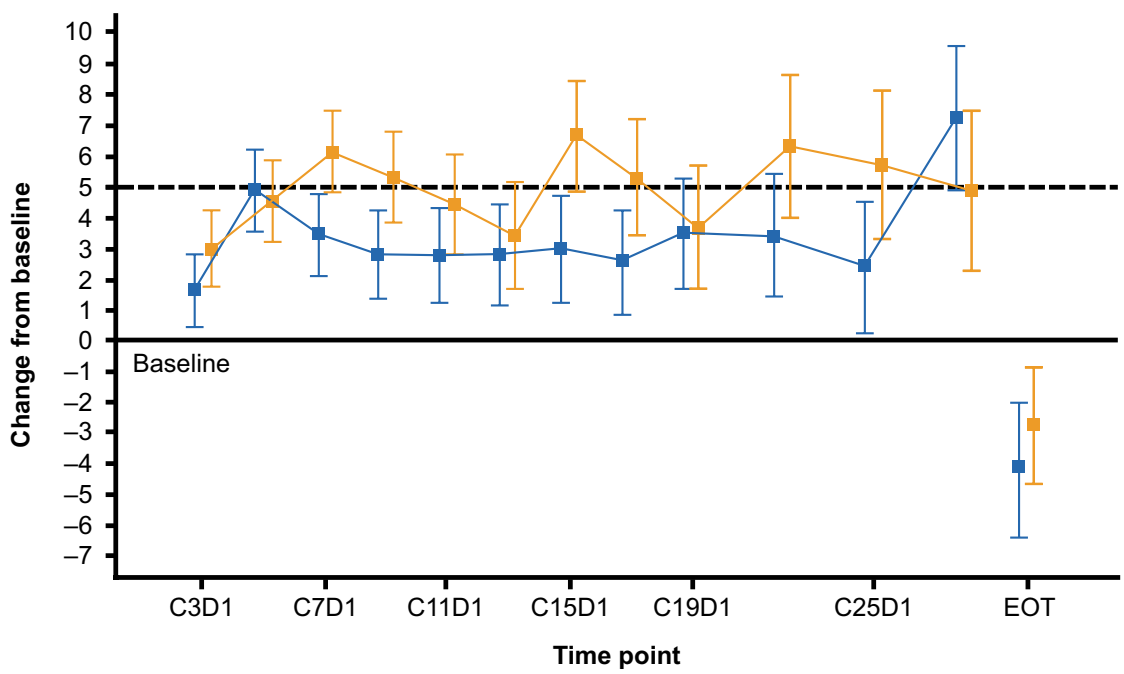

No. of patients assessed

$\begin{array}{rccccccccccccc}\text { Ribociclib + letrozole } & 178 & 161 & 156 & 143 & 141 & 132 & 120 & 116 & 104 & 92 & 85 & 56 & 91 \\ \text { Placebo + letrozole } & 175 & 156 & 151 & 136 & 118 & 115 & 104 & 93 & 84 & 68 & 67 & 45 & 123\end{array}$

(b)

$\mathrm{LSM} \pm \mathrm{SEM}$

Threshold for clinically meaningful improvement from baseline

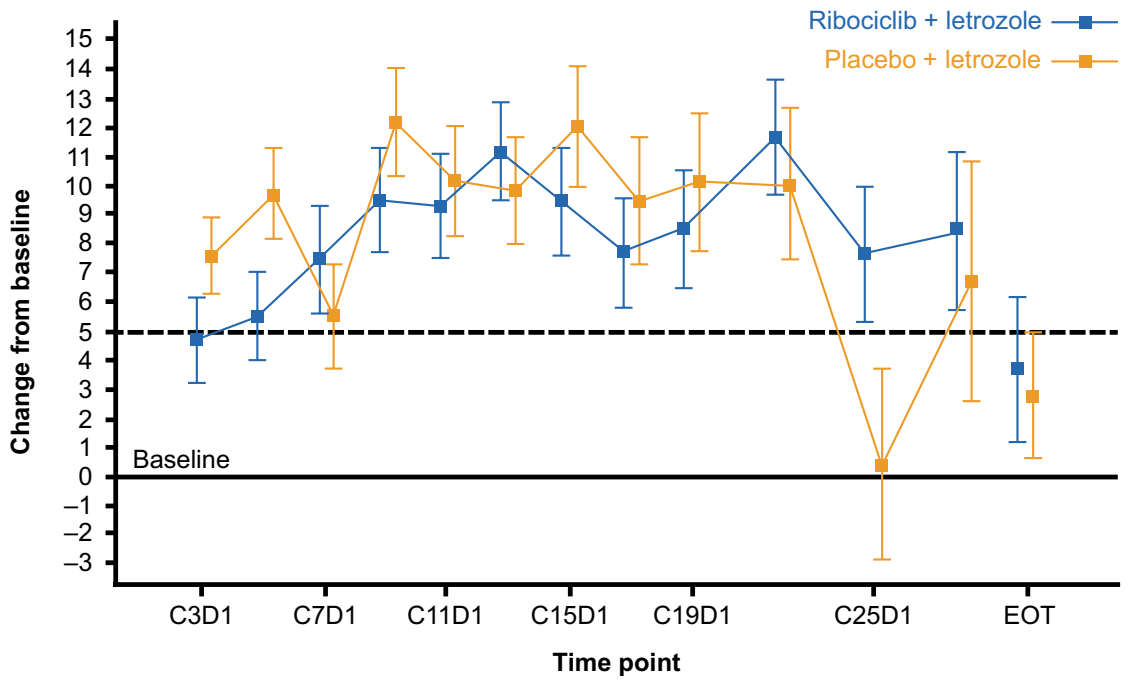

No. of patients assessed

$\begin{array}{rlllllllllllll}\text { Ribociclib + letrozole } & 107 & 101 & 93 & 89 & 82 & 73 & 69 & 64 & 57 & 59 & 54 & 31 & 57 \\ \text { Placebo + letrozole } & 110 & 98 & 97 & 83 & 75 & 65 & 57 & 47 & 43 & 35 & 28 & 15 & 78\end{array}$

Fig. 2 Overall change from baseline in patient-reported EORTC QLQ-C30 global health status/QoL scores in patients with a baseline ECOG PS of 0 (a) or 1 (b). C Cycle, D Day, ECOG PS Eastern Cooperative Oncology Group performance status, EORTC QLQ-C30 European Organisation for Research and Treatment of Cancer core quality-of-life cancer questionnaire, EOT end of treatment, HRQoL health-related quality of life, $L S M$ least square mean, $Q o L$ quality of life, SEM standard error of the mean. Changes from baseline in patient-reported EORTC QLQ-C30 global health status/QoL scores were determined using a linear mixed-effect model. Positive changes from baseline indicate improvement in HRQoL. A > 5-point improvement from baseline in HRQoL score was defined as clinically meaningful. Data cut-off: January 4, 2017 
Fig. 3 Time to definitive deterioration of global health status/QoL scale score of EORTC QLQ-C30 from baseline by $\geq 10 \%$ in patients with or without a PFS event (disease progression or death) in the ribociclib plus letrozole arm (a), placebo plus letrozole arm (b), and in all treated patients across both arms (c). CI confidence interval, EORTC QLQ-C30 European Organisation for Research and Treatment of Cancer core quality-of-life questionnaire, $P F S$ progressionfree survival, $Q o L$ quality of life, TTD time to definitive deterioration. Data cut-off: January 4, 2017 (a)

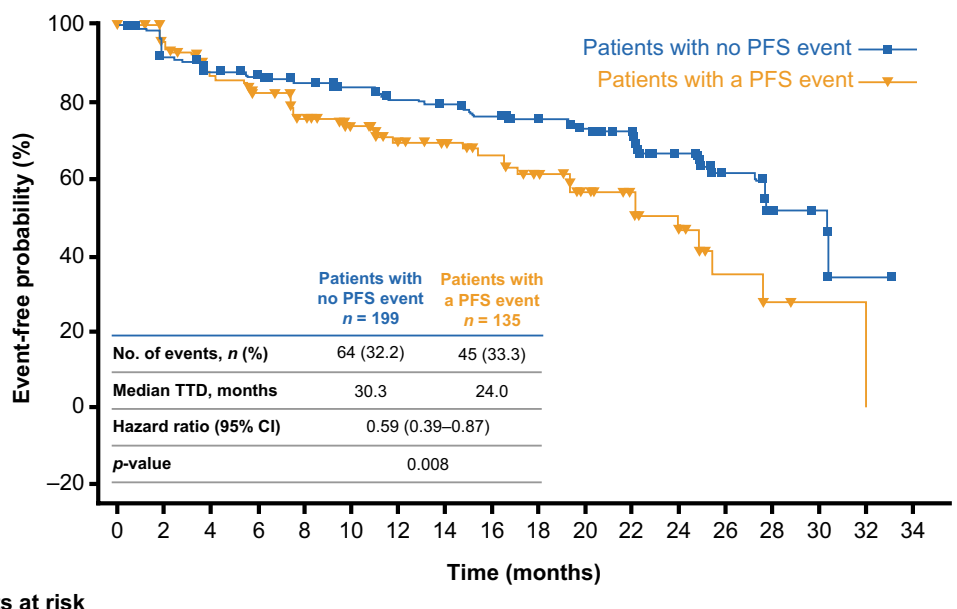

\section{No. of patients at risk}

No progression/death $199168157152145137129126120113106101 \quad 66 \quad 32 \quad 11 \quad 9 \quad 1 \quad 0$

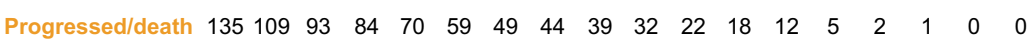

(b)

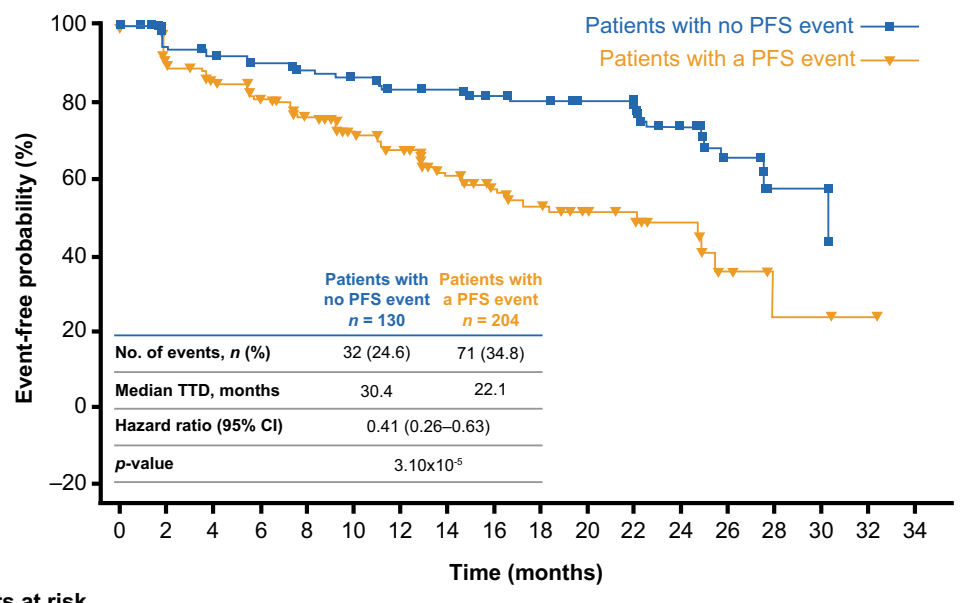

\section{No. of patients at risk}

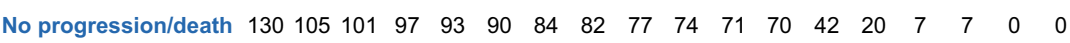

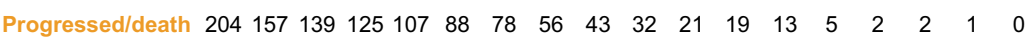

(c)

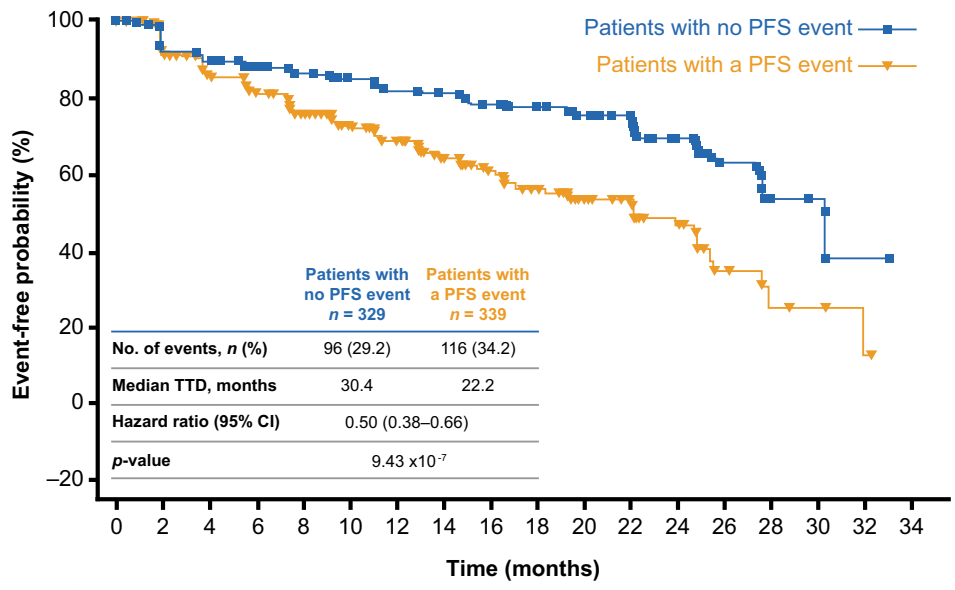

No. of patients at risk

No progression/death $329273258249238227213208197187177171108 \quad 52 \quad 18 \quad 16 \quad 1 \quad 0$

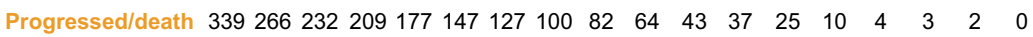


Table 3 Future perspective, side effects, and upset by hair loss scores of EORTC QLQ-BR23-mean score by treatment and visit

\begin{tabular}{|c|c|c|c|c|c|c|}
\hline \multirow{2}{*}{$\begin{array}{l}\text { EORTC QLQ- } \\
\text { BR23 mean score }\end{array}$} & \multicolumn{2}{|c|}{ Future perspective } & \multicolumn{2}{|l|}{ Side effects } & \multicolumn{2}{|c|}{ Upset by hair loss } \\
\hline & $\begin{array}{l}\text { Ribociclib + } \\
\text { letrozole } \\
n=334\end{array}$ & $\begin{array}{l}\text { Placebo + } \\
\text { letrozole } \\
n=334\end{array}$ & $\begin{array}{l}\text { Ribociclib }+ \\
\text { letrozole } \\
n=334\end{array}$ & $\begin{array}{l}\text { Placebo + } \\
\text { letrozole } \\
n=334\end{array}$ & $\begin{array}{l}\text { Ribociclib + } \\
\text { letrozole } \\
n=334\end{array}$ & $\begin{array}{l}\text { Placebo }+ \\
\text { letrozole } \\
n=334\end{array}$ \\
\hline Baseline & 41.2 & 42.2 & 14.6 & 15.2 & 15.4 & 19.2 \\
\hline Cycle 3 Day 1 & 49.2 & 51.7 & 21.3 & 17.7 & 27.7 & 30.2 \\
\hline Cycle 5 Day 1 & 54.0 & 55.4 & 20.8 & 17.8 & 34.1 & 29.6 \\
\hline Cycle 7 Day 1 & 53.6 & 57.1 & 20.7 & 17.5 & 37.5 & 33.3 \\
\hline Cycle 9 Day 1 & 56.2 & 59.7 & 21.2 & 17.2 & 39.5 & 35.6 \\
\hline Cycle 11 Day 1 & 54.2 & 58.6 & 21.4 & 17.8 & 42.0 & 33.3 \\
\hline Cycle 13 Day 1 & 58.4 & 59.7 & 21.6 & 18.2 & 36.0 & 34.5 \\
\hline Cycle 15 Day 1 & 58.4 & 64.0 & 21.7 & 16.2 & 39.2 & 30.2 \\
\hline Cycle 17 Day 1 & 58.3 & 64.0 & 20.8 & 17.6 & 36.8 & 35.4 \\
\hline Cycle 19 Day 1 & 58.3 & 63.5 & 21.1 & 16.9 & 34.4 & 27.8 \\
\hline Cycle 22 Day 1 & 63.5 & 62.7 & 20.7 & 17.1 & 34.5 & 30.6 \\
\hline Cycle 25 Day 1 & 57.8 & 64.2 & 21.3 & 17.0 & 30.7 & 26.7 \\
\hline EOT & 44.1 & 46.7 & 24.1 & 19.7 & 37.9 & 30.6 \\
\hline
\end{tabular}

EORTC QLQ-BR23 European Organisation for Research and Treatment of Cancer breast cancer-specific questionnaire, EOT end of treatment

Only time points with data available for at least 35 patients in each treatment arm are included

For future perspective, a score of $0=$ worst and a score of $100=$ best. For side effects and upset by hair loss, a score of $0=$ best and a score of $100=$ worst. A 5-10-point improvement from baseline in EORTC score was defined as clinically meaningful

Fig. 4 Change from baseline in EORTC QLQ-C30 pain scores by treatment arm. $C$ Cycle, D Day, EORTC QLQ C30 European Organisation for Research and Treatment of Cancer core quality-of-life questionnaire, EOT end of treatment, $L S M$ least squares mean, SEM standard error of the mean. Changes from baseline in patient-reported EORTC QLQ-C30 pain scores were determined using a linear mixed-effect model. Negative changes from baseline indicate a reduction in pain. $\mathrm{A}>5$-point change from baseline in pain score was defined as clinically meaningful. Data cut-off: January 4, 2017

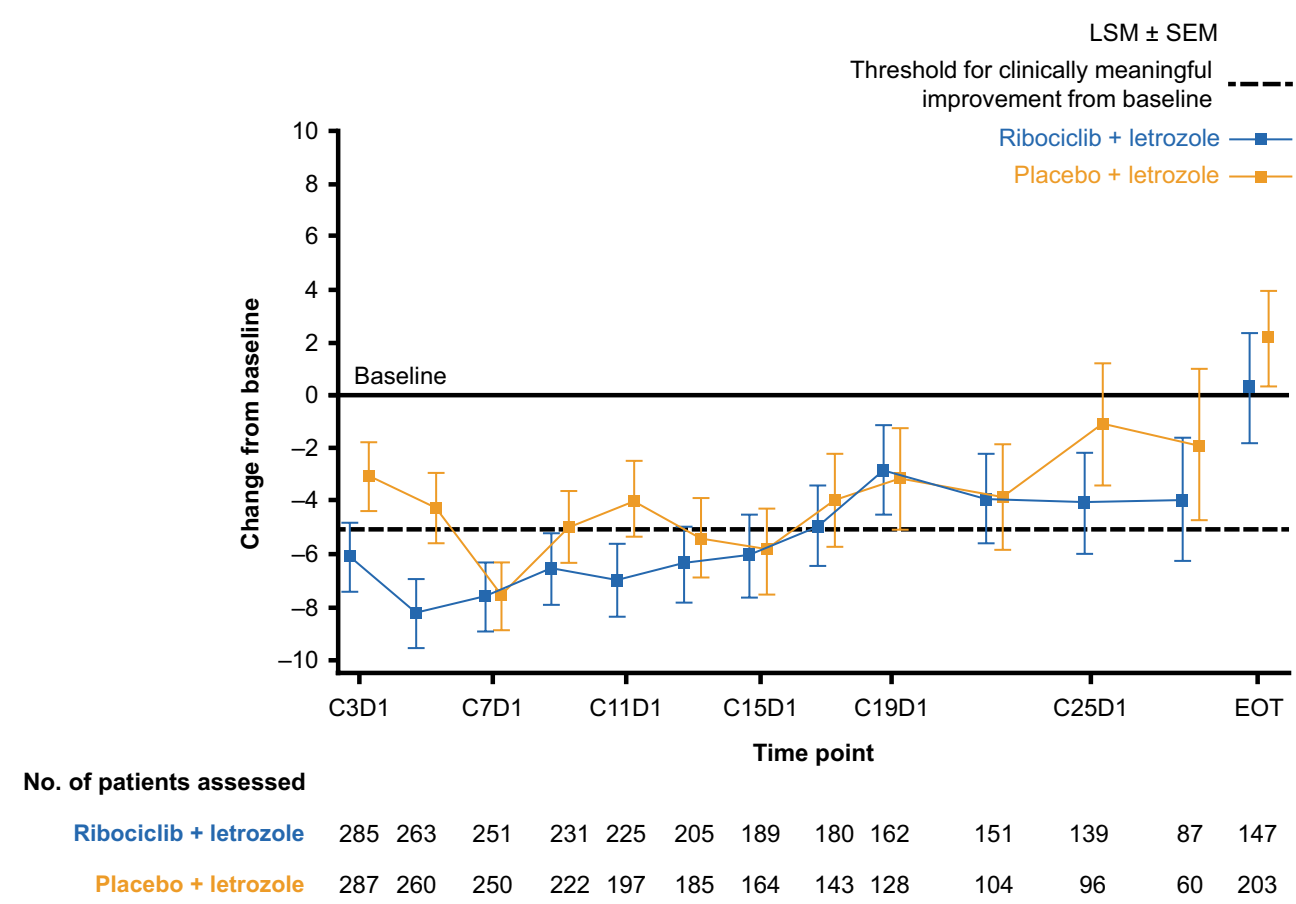




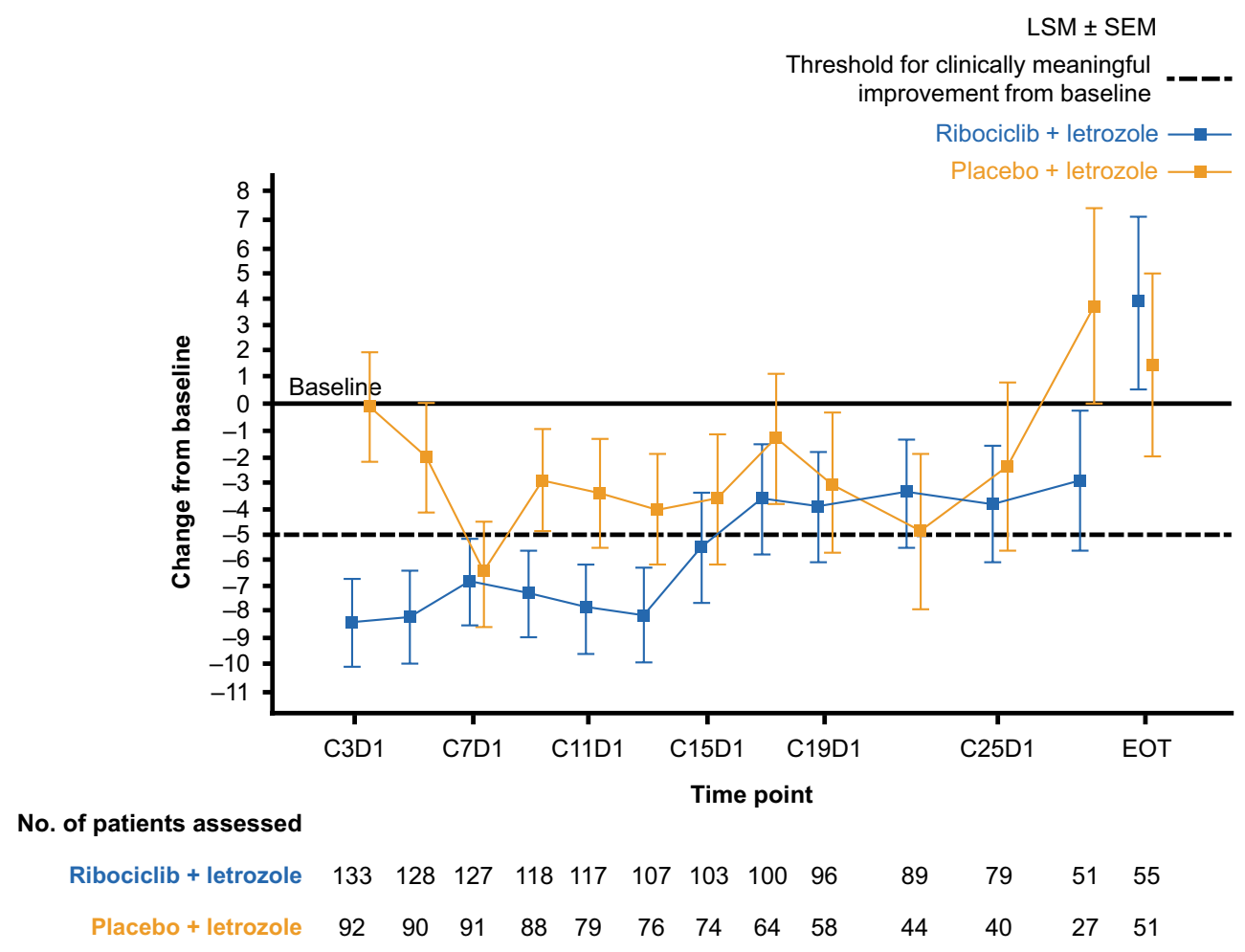

Fig. 5 Change from baseline in EORTC QLQ-C30 pain scores in patients with a best overall response of CR or PR. $C$ Cycle, $C R$ complete response, D Day, EORTC $Q L Q-C 30$ European Organisation for Research and Treatment of Cancer core quality-of-life questionnaire, $E O T$ end of treatment, $L S M$ least squares mean, $P R$ partial response,

impact on QoL [18]. However, with recent advances in the treatment of metastatic/recurrent breast cancer, preserving patient QoL has become more manageable due to the availability of more tolerable agents, such as hormone therapy and CDK4/6 inhibitors $[13,19]$. In addition to significantly improved efficacy with ribociclib plus letrozole versus placebo plus letrozole [4, 14], the current MONALEESA-2 analysis further demonstrates that ribociclib plus letrozole does not compromise patient QoL. HRQoL was maintained throughout the study treatment period in patients receiving ribociclib plus letrozole, but rapidly declined in both treatment arms at EOT, suggesting that HRQoL worsened in line with disease progression. In addition, a significantly greater delay in TTD in HRQoL was observed in patients without, versus with, a PFS event, suggesting that a delay in progression may help delay deterioration in HRQoL. One possible limitation of our study is the limited PRO measurement postprogression, which could have provided further insights on the impact of disease progression on HRQoL. In addition, considering the longer PFS in the ribociclib plus letrozole arm, the follow-up duration for PROs for these patients was likely to be longer versus the placebo plus letrozole arm. Despite the potential difference in follow-up, evaluation of
SEM standard error of the mean. Changes from baseline in patientreported EORTC QLQ-C30 pain scores were determined using a linear mixed-effect model. Negative changes from baseline indicate a reduction in pain. A $>5$-point change from baseline in pain score was defined as clinically meaningful. Data cut-off: January 2, 2017

PROs postprogression may reveal more pronounced HRQoL differences between arms and in patients with or without progression events.

In addition to maintaining overall QoL, ribociclib plus letrozole was associated with a clinically meaningful reduction in pain in the overall population, which was observed as early as Week 8 and maintained for at least 15 cycles. Significant improvements in pain score were also observed in all patients and subgroups of patients with measurable disease at baseline in the ribociclib plus letrozole arm following an exploratory AUC analysis. In a cross-sectional study involving 1072 patients with breast cancer, maintaining QoL and controlling pain were among the top 10 most important issues [20]. In addition, increasing pain severity has been associated with significant worsening of QoL in patients with advanced cancer [21]. Current guidelines suggest that assessment and management of pain is of critical importance in patients with cancer but is not adequately treated, despite recommendations that effective pain management be included as part of the treatment plan $[9,22]$. Given that pain adversely impacts QoL, reducing or delaying pain symptoms could be expected to improve HRQoL. In light of this, the early 


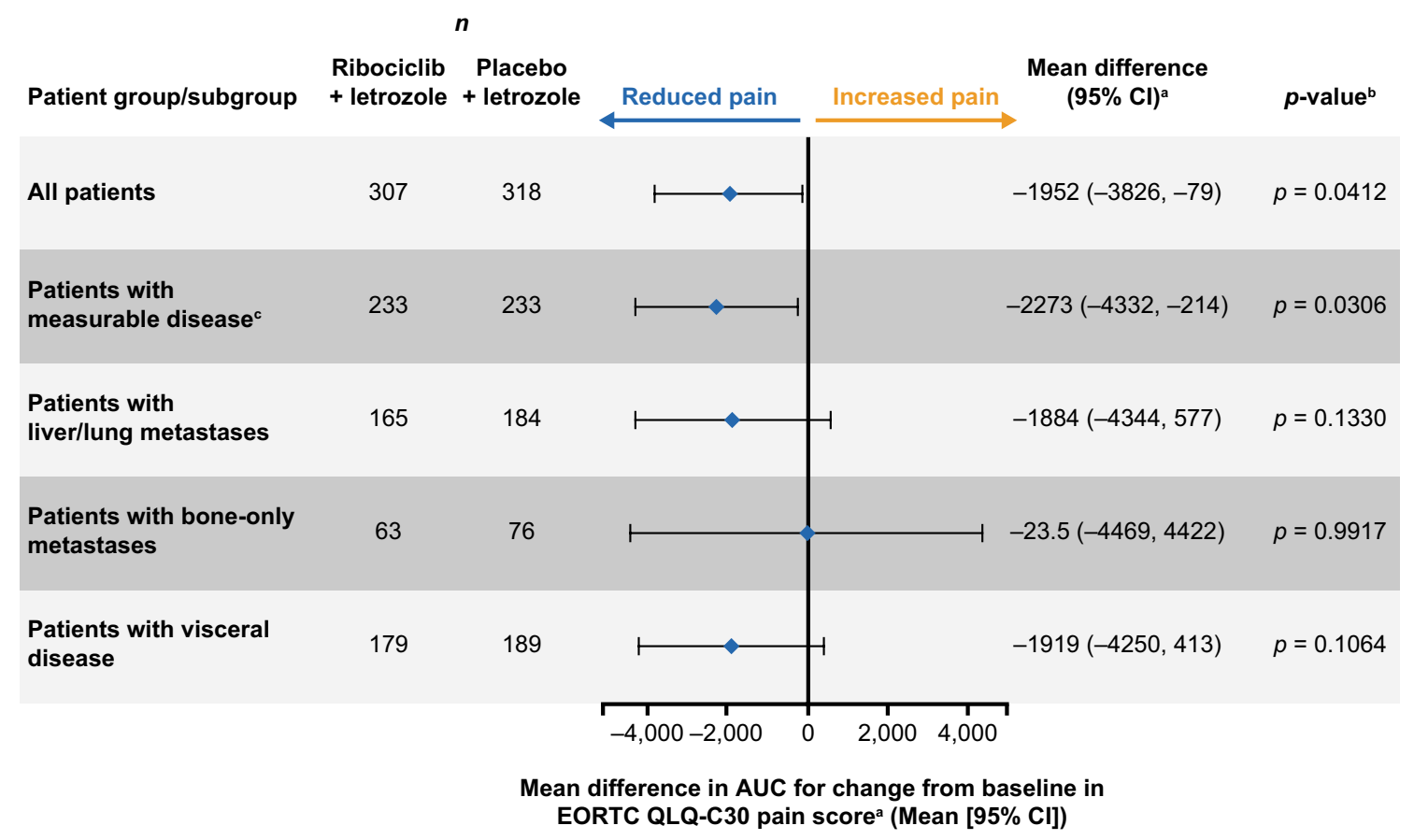

Fig. 6 Exploratory AUC analysis for the mean difference in change from baseline in EORTC QLQ-C30 pain scores between treatment arms. AUC area-under-the-curve, CI confidence interval, EORTC QLQ-C3O European Organisation for Research and Treatment of Cancer core quality-of-life questionnaire. AUC analysis for change from baseline in mean EORTC QLQ-C30 pain scores was performed for the indicated subgroups in each treatment arm. Larger negative

improvement in pain score observed with ribociclib treatment introduces a new consideration for treatment selection in this patient population.

In conclusion, our findings demonstrated that overall HRQoL in the MONALEESA-2 study was consistently maintained from baseline in postmenopausal women with HR+, HER2- advanced breast cancer receiving ribociclib in combination with letrozole compared with placebo plus letrozole. In addition, combined ribociclib plus letrozole was associated with early and clinically meaningful improvements in pain severity compared with placebo plus letrozole. Together with the demonstrated clinical efficacy and tolerability, these PRO results provide further evidence for the benefit of ribociclib plus letrozole in this patient population.

Acknowledgements The authors would like to thank the patients who took part in this study and their families, as well as the staff at each study site, and all study steering committee members. Editorial assistance was provided by Bhavika Modasia, Ph.D., and Nicole Parker, Ph.D., of ArticulateScience Ltd. Ribociclib was discovered by Novartis Institutes for BioMedical Research in collaboration with Astex Pharmaceuticals.

Funding This study was supported by Novartis Pharmaceuticals Corporation. values indicate a greater reduction in pain. ${ }^{\mathrm{a} C o m p a r e d}$ between treatment arms using a paired $t$-test. ${ }^{\mathrm{b}} p$-values reported are nominal. No multiplicity adjustments were made, and therefore, statistical interpretation should be made with caution. ${ }^{\mathrm{c}}$ Measurable disease data were based on a data cut-off date of January 2, 2017. All other subgroup data were based on a data cut-off date of January 4, 2017

\section{Compliance with ethical standards}

Conflict of interest Dr. O'Shaughnessy has received honoraria for participating in advisory boards for Novartis outside the submitted work. Prof. Campone has participated in advisory boards for Novartis during the conduct of the study and has participated in advisory boards for Astra Zeneca, Eli Lilly, Pfizer, and Sanofi-Aventis outside the submitted work. Dr. Alba has acted in advisory roles for Novartis and Roche. Mr. Chandiwana reports stock in Novartis. Dr. Dalal reports stock in Novartis. Dr. Sutradhar reports stock or ownership in Novartis. Dr. Monaco reports stock or ownership in Novartis and Pfizer. Prof. Janni has received research grants and honoraria from Astra Zeneca, Cellgene, Chugai, Eisai, GlaxoSmithKline, Johnson \& Johnson, Novartis, Pfizer, Roche, and Sanofi-Aventis. Mr. Chandiwana, Dr. Dalal, Dr. Sutradhar, and Dr. Monaco are all employees of Novartis Pharmaceuticals Corporation. All remaining authors declare that they have no conflict of interest.

Ethical approval All procedures performed in studies involving human participants were in accordance with the ethical standards of the institutional and/or national research committee and with the 1964 Declaration of Helsinki and its later amendments or comparable ethical standards.

Informed consent Informed consent was obtained from all individual participants included in the study. 
Open Access This article is distributed under the terms of the Creative Commons Attribution 4.0 International License (http://creativeco mmons.org/licenses/by/4.0/), which permits unrestricted use, distribution, and reproduction in any medium, provided you give appropriate credit to the original author(s) and the source, provide a link to the Creative Commons license, and indicate if changes were made.

\section{References}

1. Kim S, Loo A, Chopra R et al (2013) LEE011: an orally bioavailable, selective small molecule inhibitor of CDK4/6- reactivating $\mathrm{Rb}$ in cancer. In: AACR-NCI-EORTC International Conference on Molecular Targets and Cancer Therapeutics: Abstract PR02

2. Parasuraman S, Caponigro G, Loo A et al (2014) LEE011, a potent and selective CDK4/6 inhibitor, under preclinical and clinical investigation. In: Targeted Anticancer Therapies Congress: Abstract $\mathrm{O} 4.4$

3. O'Brien N, Di Tomaso E, Ayala R et al (2014) In vivo efficacy of combined targeting of CDK4/6, ER and PI3K signaling in ER+ breast cancer. In: Proceedings of the 105th Annual Meeting of the American Association for Cancer Research: Abstract 4756

4. Hortobagyi GN, Stemmer SM, Burris HA et al (2016) Ribociclib as first-line therapy for HR-positive, advanced breast cancer. N Engl J Med 375:1738-1748

5. Hortobagyi GN, Stemmer SM, Burris HA et al (2016) First-line ribociclib + letrozole for postmenopausal women with hormone receptor-positive $(\mathrm{HR}+)$, HER2-negative (HER2-), advanced breast cancer (ABC). In: The 41st European Society of Medical Oncology Cancer Congress: Abstract LBA1_PR

6. Hortobagyi GN, Stemmer SM, Burris HA et al (2017) Updated results from MONALEESA-2, a phase 3 trial of first-line ribociclib + letrozole in hormone receptor-positive, HER2-negative advanced breast cancer. In: American Society of Clinical Oncology Annual Meeting: Abstract 1038

7. Miles D, von Minckwitz G, Seidman AD (2002) Combination versus sequential single-agent therapy in metastatic breast cancer. Oncologist 7(Suppl 6):13-19

8. Finn RS, Martin M, Rugo HS et al (2016) Palbociclib and letrozole in advanced breast cancer. N Engl J Med 375:1925-1936

9. Cardoso F, Costa A, Senkus E et al (2017) 3rd ESO-ESMO international consensus guidelines for advanced breast cancer (ABC 3). Breast 31:244-259

10. Aaronson NK, Ahmedzai S, Bergman B et al (1993) The European Organization for Research and Treatment of Cancer QLQ-C30: a quality-of-life instrument for use in international clinical trials in oncology. J Natl Cancer Inst 85:365-376

11. Sprangers MA, Groenvold M, Arraras JI et al (1996) The European Organization for Research and Treatment of Cancer breast cancer-specific quality-of-life questionnaire module: first results from a three-country field study. J Clin Oncol 14:2756-2768

12. Osoba D, Rodrigues G, Myles J, Zee B, Pater J (1998) Interpreting the significance of changes in health-related quality-of-life scores. J Clin Oncol 16:139-144

13. Verma S, O'Shaughnessy J, Burris $\mathrm{H}$ et al (2017) Health-related quality of life (HRQoL) of postmenopausal women with hormone receptor-positive $(\mathrm{HR}+)$, human epidermal growth factor receptor 2-negative (HER2-) advanced breast cancer $(A B C)$ treated with ribociclib + letrozole: results from MONALEESA-2. In: American Society of Clinical Oncology Annual Meeting: Abstract 1020

14. Janni W, Alba E, Bachelot T et al (2018) First-line ribociclib plus letrozole in postmenopausal women with HR+, HER2advanced breast cancer: tumour response and pain reduction in the phase 3 MONALEESA-2 trial. Breast Cancer Res Treat. https://doi.org/10.1007/s10549-017-4658-x

15. King MT (1996) The interpretation of scores from the EORTC quality of life questionnaire QLQ-C30. Qual Life Res 5:555-567

16. Wallwiener M, Simoes E, Sokolov AN et al (2016) Health-related quality of life in metastatic and adjuvant breast cancer patients. Geburtshilfe Frauenheilkd 76:1065-1073

17. Kovic B, Guyatt G, Brundage M et al (2016) Association between progression-free survival and health-related quality of life in oncology: a systematic review protocol. BMJ Open 6:e012909

18. Montazeri A (2008) Health-related quality of life in breast cancer patients: a bibliographic review of the literature from 1974 to 2007. J Exp Clin Cancer Res 27:32

19. Burris HA 3rd, Lebrun F, Rugo HS et al (2013) Health-related quality of life of patients with advanced breast cancer treated with everolimus plus exemestane versus placebo plus exemestane in the phase 3, randomized, controlled, BOLERO-2 trial. Cancer 119:1908-1915

20. Hollen PJ, Msaouel P, Gralla RJ (2015) Determining issues of importance for the evaluation of quality of life and patientreported outcomes in breast cancer: results of a survey of 1072 patients. Breast Cancer Res Treat 151:679-686

21. Cramarossa G, Chow E, Zhang L et al (2013) Predictive factors for overall quality of life in patients with advanced cancer. Support Care Cancer 21:1709-1716

22. Ripamonti CI, Santini D, Maranzano E et al (2012) Management of cancer pain: ESMO clinical practice guidelines. Ann Oncol 23(Suppl 7):vii139-vii154 